\title{
Strategic maintenance-management in Nigerian industries
}

\author{
Mark C. Eti ${ }^{*}$ S. O. T. Ogaji ${ }^{* *+}$ and S. D Probert ${ }^{* *}$ \\ *Mechanical Engineering Department, Rivers State University Of Science and Technology, \\ PMB 5080 Nkpolu, Oroworukwo, Port Harcourt, Rivers State, Nigeria \\ ** School Of Engineering, Cranfield University, Bedfordshire MK43 OAL, United Kingdom. \\ ${ }^{+}$Corresponding author
}

Keywords: strategy; maintenance; Nigerian electric-power stations

\begin{abstract}
:
A developing society needs to adapt to change and foster creativity. In the pursuit of continual improvement (i.e. reducing energy consumption, better service performance, greater availability and improved reliability), implementing wise maintenance schedules is essential for contemporary organisations. Several studies of a wide range of Nigerian industries indicate that indigenous low availability and low productivity are endemic. The resulting closure of some of these industries has triggered off a realisation of the strategic challenges in maintenance management. In addition, the contemporary increasingly-competitive business environment in Nigeria has raised the strategic importance of maintenance functions, especially in organisations with significant investments in physical assets. Five strategic aspects of maintenance management have been identified, namely: - maintenance methodology; support processes; organisation and work structuring; comparable culture; and general management. Three factors that permeate these dimensions are wise leadership, excellent communications and an understanding of the human factors involved.
\end{abstract}

\section{Abbreviations}

$\begin{array}{ll}\text { ASP } & \text { Application service provider } \\ \text { BSC } & \text { Balanced score card } \\ \text { CBM } & \text { Condition-based maintenance } \\ \text { CEO } & \text { Chief executive officer } \\ \text { CMMS } & \text { Computerised maintenance management system } \\ \text { ES } & \text { Enterprise system } \\ \text { FMECA } & \text { Failure modes, effect and critical analysis } \\ \text { JIT } & \text { Just-in-time } \\ \text { OEE } & \text { Overall equipment effectiveness } \\ \text { RCM } & \text { Reliability-centred maintenance } \\ \text { SMPM } & \text { Strategic maintenance performance management } \\ \text { SMT } & \text { Self-management team } \\ \text { STS } & \text { Socio-technical systems } \\ \text { TPM } & \text { Total productive maintenance } \\ \text { TPQM } & \text { Total planned quality maintenance } \\ \text { TQM } & \text { Total quality management }\end{array}$

\section{THE CHALLENGE}

The subject of change influences much of what is currently being written about management. All organisations are being exhorted to adapt to changes and hence introduce modern technology, leadership skills and more effective means of communication. It has become 
evident that any organisation that wishes to achieve rapid, substantial and sustainable improvements in maintenance effectiveness, in other words in physical-asset performance, must act strategically.

Maintenance actions are dependent on such factors as the plant's previous downtime history, and its expected life. Maintenance should preserve the functions of the assets. With rising expectations, increasingly onerous regulatory constraints, shifting technological paradigms and frequent reorganisations, major corporations have developed formal mission-statements to help them maintain a successful course through these many demands. Thus, it is worth developing a corresponding commitment to facilitate the maintenance team doing likewise. The mission statement must the recognize needs of all the stakeholders of the maintenance service. Maintenance serves three distinct groups - the owners of the asset, the users (usually the operators), and society as a whole. Maintenance depends on a range of people - from designers of the equipment to its operators. Thus it is desirable to acknowledge the need for everyone involved with the asset to share a correct common understanding of what needs to be done, and to be able and willing to do whatever is needed right first time, every time.

If systems do not fail, maintenance would be superfluous. The technology of maintenance includes finding and applying suitable ways of managing techniques that include predictive and preventive maintenance, failure-finding and run-to-failure [1]. Each category includes a host of options: maintainers need to learn what these options are, but they also need to decide which are worth implementing in their own organisations. If they make the right choices, it is possible to improve asset performance, and simultaneously even reduce the cost of maintenance. If they make wrong choices, new problems may arise while existing problems get worse, so the mission statement should stress the need to make the most cost - effective choices from the full array of options. The severity and frequency with which a failure incurs adverse consequences dictate whether a particular failure-management technique is worth applying. So, the mission statement should acknowledge the key role of consequence avoidance in maintenance. The development and execution of a maintenance strategy for an industrial plant consists of three steps [1]: -

- Formulate a maintenance strategy for each asset (i.e. work identification)

- Acquire the resources (namely skilled people, spares and tools) needed to execute the strategy effectively

- Execute the well thought-out strategy (i.e. deploy and operate the systems needed to manage the resources efficiently).

Most of what has been written on maintenance strategy refers to the three basic types namely, predictive, preventive and corrective maintenance. However, Moubray [2] adds a fourth, that is, detective maintenance. Predictive (or condition-based) tasks entail checking to see if the process is failing. Preventive maintenance usually means overhauling a system before its failure occurs. Preventive maintenance is divided into time-based and condition-based maintenance. Time-based (according to a fixed time schedule) maintenance is wasteful if no faults exist. Corrective maintenance means fixing or replacing components either when they are found to be failing or when they have failed. Detective (i.e. failure-finding) maintenance applies only to hidden or unrevealed failures and hidden failures usually only affect protective devices.

In spite of the availability of all of the afore mentioned maintenance policies and their abilities to prevent failures or restore the system to its operating condition in most situations, deteriorations and breakdowns still occur and associated environmental disasters happen. Each maintenance policy has its own limitations and disadvantages. For example, in the case 
of failure-based maintenance, it is difficult to plan the maintenance activities appropriately: thus, the failure of the considered component can cause a large amount of consequential damage to other parts of the system or plant. Time-based maintenance incurs a large financial cost for the user in trying to maintain the required level of system reliability, because the majority of items are replaced prematurely, i.e. while they still have a considerable useful-life remaining [3].

In condition-based maintenance, it may not be cost effective to monitor the condition of every component of the system: some will be inaccessible for monitoring. So, it is beneficial not to treat condition-based maintenance as a stand-alone policy, but instead as a part of an integrated maintenance strategy. Consequently, the industry has become aware that a single maintenance policy cannot eliminate all breakdowns or restore the plant to its full operating capability. This awareness gave birth to maintenance strategies like reliability-centred maintenance (RCM) and total productive maintenance (TPM).

(i) RCM determines what must be done to ensure that a physical asset continues to do whatever it was designed to do. A failure of one component may stop a whole plant performing to the standard required by its users. Before any blend of failure-management tools can be applied successfully, it is necessary to identify what failures are likely to occur. The RCM process: -

- Identifies what circumstances amount to a failed state.

- Asks what event can cause the asset to fail.

- Provides a maintenance-oriented framework to meet the challenges.

- Deals effectively with each type of failure process with appropriate maintenance tactics.

- Improves maintenance productivity by adopting a more proactive, planned approach.

- Extends the run duration between scheduled shutdowns.

- Ensures the active support and cooperation of all personnel, e.g. between the maintenance, materials, operations and technical functions.

The RCM process develops and optimises the effective maintenance programme.

(ii) TPM: RCM is an asset-centred methodology with the primary focus on making decisions concerning the type of maintenance tasks to be used, whereas TPM is a methodology with a different orientation - it focuses on people and is an integral part of TQM [4]. TPM methodology was developed initially in Japan's manufacturing industries, with the aim of reducing production losses due to machine breakdowns in JIT production systems.

TPM organises maintenance procedures by applying the following [4]:

- Cultivate a sense of ownership in the operator by introducing autonomous operator maintenance i.e. the operator takes responsibility for the primary care of his/her plant. The tasks involved include routine inspection, lubrication, adjustment, major repairs, as well as cleanliness and tidiness of the operator's workspace.

- Optimise the operator's skills and knowledge of his/her plant to maximise the operating effectiveness. The operator is thus mobilised to detect any early signs of wear, maladjustment, oil leak, or loose parts. He/she is also involved in making improvement suggestions to reduce the losses due to a break-down or sub-optimal performance of the plant. 
- Uses cross-functional teams, consisting of operators, maintenance technicians, engineers and managers to improve performances of those members and equipment.

- Establish a schedule of clean -up and preventive maintenance to extend the plant's life-span and maximizes its up-time.

\section{PRACTICAL IMPLICATIONS}

In many industries worldwide, the effects of downtime are becoming aggravated by the increasing adoption of just-in-time (JIT) processes and total quality-management (TQM). Reduced stocks associated with JIT mean that minor breakdowns are now much more likely to stop or inhibit production. The growth of mechanisation and automation means that reliability and availability have become key issues in sectors as diverse as healthcare, electricpower generation stations and chemical processing. Thus, during recent decades, the cost of maintenance has risen in absolute terms and as a proportion of total expenditure. In some industries, it is now the largest contributor to the total cost. Maintenance reactions are dependent on many factors, such as failure rates of the plant's components, the associated costs of downtime and the expected lives of critical items of equipment. Maintenance strategies have evolved (in highly industrialised countries) taking into account quality, cost, flexible delivery and customer satisfaction. Maintenance is regarded as a proactive profitfocused activity; the aim being to narrow the difference between actual and ideal maintenance costs, i.e. reduce the cost of unreliability. Recent developments [4] are: -

- Emerging operational strategies. The economy-of-scale concept is losing some of its advocates. Increasingly, organisations have switched to lean manufacturing, "JIT production" and "six - sigma programmes". These trends indicate a shift of emphasis from:- maximum volume output to quick response; elimination of waste, defect prevention; and achievement of higher quality. In increasingly demanding environments, breakdowns, speed loss and erratic process yields create immediate problems for the timely supply of goods or services to customers. Thus, installing the appropriate equipment and facilities, optimising the maintenance schedules of these assets and the effective deployment of appropriately skilled manpower to perform the maintenance activities are crucial factors in fostering these operational strategies.

- Raised societal expectations. There is a growing acceptance in theory (but too often not in practice) of the need to protect the ambient environment as well as ensure people's safety and health. In the developed countries, wide ranges of regulations have been enacted to control industrial-pollution and prevent accidents occurring in the work place. Scrap, defects and the ineffective uses of materials and energy are recognised as sources of pollution. Nevertheless, catastrophic failures of operating plant, industrial accidents and health hazards are still far too prevalent. Keeping facilities operating optimally and preventing failures are means of meeting, partially, the ever more demanding societal challenges with respect to reducing pollution, emission and accident prevention. These should now be core functions in any maintenance programme, but too rarely are.

- Technological changes. Technology is a major driver of change. In the electricitygenerating industry, automation systems are being installed to identify and deal with faults in the transmission and distribution network. New technologies are being deployed to enhance the system's availability, raise the cost effectiveness of all 
operations and deliver better or innovative services to customers. These present new challenges to maintenance function.

- Changes in personnel attitudes and organisational systems. Most personnel wish to improve their quality-of-life at work. Furthermore, the social and demographic changes that have taken place recently affect how we regard and define work. Two examples of these changes are (i) improvements in pertinent education and (ii) increased faith in the ability of individuals to manage them well in the face of adversity. Thus, progressive organisations have introduced less hierarchical management structures, self-managing work-teams, virtual organisations and strategic alliances. Some of these could be appropriate for meeting today's challenges of providing improved maintenance services to organisations.

\section{ORGANISATION AND WORK STRUCTURING}

Tsang [4] concluded that the strategic decisions to be taken with respect to the organisational design and structuring of maintenance activities include: (i) plant purpose, (ii) workforce location, and (iii) composition and flexibility of their workforce. The associated decisions require considering workload characteristics; plant location; cost of non-availability; skills and knowledge required; production policy; and human-resources policy. In traditional Nigerian organisations, the hierarchical management structure is often parochial and highly functionalised: e.g., engineering is responsible for the design and procurement of new plant as well as the modification of existing systems; production is responsible for operating the plant; and maintenance for servicing and repairing it. Furthermore, the maintenance workforce is organised into specialised trades, so resulting in demarcation disputes, lack of cooperation and poor operational efficiencies. Although multi-skilling has often been successful in creating a more flexible work-force, experience now highlights that such employees often lose the motivation to seek out solutions to basic equipment problems or defects (which, if left unchecked, will subsequently cause failures) because they know they will soon be moved to another task or to attend to a different piece of equipment. A zone-based team approach, which promotes the development of both base-skills and interdisciplinary-skills, provides a means to achieve both flexibility of being capable of responding effectively and ownership (of the problem) within the workplace. Wisely formed zone-based teams create a learning environment, where employees recognise the self-benefits of being able to operate, and care for, their equipment [5].

The workforce may range from a plant-flexible tradesman, who is responsible for maintaining all the plant to a plant-specialized one, who only works on a particular type of equipment. The advantages of plant specialization include improved work-quality and faster responses due to the greater plant knowledge and sense of ownership. A stronger spirit of teamwork can also develop. However, this mode of organization will face a major problem when the workload varies considerably from one plant-specialised group to another. In such cases, labour utilization will be lower because the workload cannot be balanced by exploiting labour mobility.

Flexible trade-groups are usually located centrally or close to their designated zone. The main problem with decentralized plant-specialized groups is that it is difficult to achieve flexible labour movements between trade groups. The knowledge and skills involved determine how the maintenance function is organised. The degree of centralization of the maintenance function can also be an indication of an assets' life-cycle. 
Maintenance work usually requires a range of skills in any operation, although a single skill is nevertheless predominant for that operation. If the operation requires (i) special skills and (ii) the workload is relatively constant with respect to time, it is appropriate to adopt trade specialization [4].

As a result of the adoption of work structuring, organisations tend to evolve from rigid functionalism towards task - based structures. Taylor and Felton [6] provided a theoretical basis for socio-technical system (STS) concepts, e.g. self-managing teams (SMTs). Holodny and Stjeinberg [7] proposed the following design principles for establishing SMTs:

- Team activities are task oriented and designed with a performance focus.

- The team is organised to perform whole, integrated tasks.

- The team should have some autonomy (that is, control over many of its own administrative functions, such as self-planning, self-evaluation and self-regulation). Furthermore, members should participate in the selection of new team-members.

- Detailed specifications of tasks, procedures and methods are kept to a minimum. Only those essential for information sharing with outside parties, for scheduling or for coordination are prescribed. Within the team, standards are arrived at through agreement about what represent group norms. This flexibility allows the team to evolve as members develop and increase their multi-skilling competencies.

- Being multi-skilled is highly valued. This approach fosters the development of confidence amongst team members: they become more able to cope with unanticipated events.

\section{MAINTENANCE CULTURE AND LEARNING}

A dictionary definition of culture is that it encompasses the inherited ideas, beliefs, values and knowledge, which constitute the shared basis for social action. Culture is an integrated pattern of human behaviour and interactions. This behaviour includes thought, speech, actions, and those objects created as a result of our actions [8]. Culture endures and evolves through our capacity for learning and consequently, through sharing current knowledge with succeeding generations. Hence, culture is the total of the inherited ideas, beliefs, values and knowledge that contribute the shared bases of social actions. The required behaviour only becomes part of the culture through learning and through validation as being acceptable and desirable by the majority of the organisation.

Business environments need to reflect the ways that the involved personnel wish to live. Most enlightened companies have at least heard of a work/life balance, even if they do not actively promote it among their staff. It makes sense to provide an environment and a culture, which contributes to, rather than drains the satisfaction from their staff's personal lives. Each organisation should be committed to creating a culture of support, growth, responsibility and vision. Considering staff as individuals, rather than as a generic workforce, is now seen by most companies as a pre-requisite to achieving a positive company culture and hence a higher productivity.

Today's gurus of strategy urge companies to democratise the management process, which was once the sole province of a company's senior officers, by handing strategic planning over to teams of line and staff managers from different disciplines. To keep the planning process 
close to the reality of the market, today's strategists say it should also include interaction with key customers and suppliers.

It is important that organisations, and particularly top management of those organisations, plan effectively and define their ideals, and in particular, state what are the norms and minimum values, which support the defined strategy. Top management expresses its ideals through vision, mission, value and policy statements: it is common for these to include references to people as being valuable assets. Notwithstanding, only very few organisations have proactive programmes to manage cultural aspects.

From a maintenance perspective, top management is probably the most significant influence on the maintenance culture. This echelon should create and maintain an internal environment in which the personnel can become fully involved in achieving the organisation's objectives. Organisations that take management systems seriously develop feedback, monitor prevailing achievements culture and compare them with the ideal.

Most organisations that do not devote sufficient attention to their human capital, particularly in the measurement of quality and effectiveness, usually under-utilise the talent of their workforces. So it is wise for senior managers to invest in assessing the effect of human-capital management on profitability to enable the development and establishment of best-practice guidance that will help the organisation put the necessary tools in place. The aim is to develop a culture that supports the organisation's values while achieving the targeted business results. Each organisation should be able to compare its devised culture and by using focus groups, propose relevant action plans. There must be an assessment of the attitudes, mind-sets, and beliefs that determine if the maintenance process is accomplished well. Without an appropriate culture, it will be difficult to identify what is acceptable day-by-day behaviour. A learning culture fosters personnel, so that they become more innovative and creative by harnessing their diverse and individual abilities. Developing the required culture should be considered to be an integral part of the whole management process. To remain internationally competitive, firms must sustain a high rate of internal learning that both refines current practices and adopts better ones. Government agencies are continually being required to provide a broader range of services faster, yet without using any extra resources [9]. External benchmarking programmes are popular ways of identifying good practices and show the benefits of demonstrating what is realistically achievable. The learning processes involved in establishing and implementing such programmes also provide benefits for those involved in implementing them. Drew [10] identified several successes achieved by various forms of benchmarking, but also pointed out some potential barriers. According to Harvey and Denton [11], six factors are leading to the growing popularity of organisational learning:-

- The increasing importance of achieving higher productivities.

- The accelerating pace of change in the business environment .

- Pertinent knowledge viewed as a means of gaining a competitive advantage.

- Customers becoming more demanding.

- Dissatisfaction with the existing management paradigm.

- The increasing intensity of competition in the global market

Successful learning needs time and patience. According to Senge [12], a discipline is a body of practices, based on an underlying theory: it suggests the appropriate path of development. The five disciplines are (i) building a shared mission, (ii) personal mastery, (iii) working with mental models, (iv) team learning and (v) system thinking. The first step in creating a learning 
environment should be leadership that is based on teaching abilities, and which assists others in understanding complex situations [13].

\section{MANAGEMENT}

In today's businesses, the achievement of success usually requires the ability to change and adapt quickly. Organisational change is seen as a way of addressing outdated styles of management and practices. The key to implementing effective organisational change is the wise management of the process (whether it be for cultural change, strategic and business planning, process design, management development or performance improvement). Globalisation of the market and the rapid rise in information-flow capabilities have increased competition worldwide. In order to compete successfully in todays turbulent, highly competitive business-environment, organisations are focusing on the satisfaction of customers' needs as a means of securing a competitive advantage, or even survival. A major strategic change is that many organisations are striving to achieve customer satisfaction through an increased emphasis on quality in their products and services.

TQM has become an umbrella philosophy that encompasses all aspects of management ranging from strategy, tactics and cost reduction to customer satisfaction and employee empowerment. The rigorous implementation of TQM has resulted in a new workplace culture and led to changes in management and processes. TQM's philosophy requires the reduction of costs yet an improvement in the quality of goods produced and/or services provided as well as increased flexibility and responsiveness. Operations strategies are often focused on developing strategic alliances based on core competencies. In the information age, companies tend to operate in a physically-distributed manufacturing or service environment. To integrate the activities along the value chain, various information technologies have been used, including appropriate education and training to deal with diversities in language and culture see for instance the Japanese 5s principles, the 1S0 9001: 2000 standards and the six-sigma criteria, whose implementation will help achieve TQM. The ' $5 \mathrm{~s}$ ' stands for sort (organise), set in order (orderliness), shine (cleanliness), standardise and sustain (through applying discipline): this set of principles enables one to realise what is possible and what can be done realistically to improve quality. TQM on the other hand delivers a behavioural mindset and a belief in the achievement of continual improvement that are essential for making concepts such as six-sigma work well.

Skills in 'change' management enable personnel to build trust and constructive relationships with their colleagues: in particular, persuasive communications, inspiring motivations and leadership skills are desirable. Excellent leadership can be achieved through having a clear vision of what is needed, a well-understood, comprehensive mission statement agreed by all personnel, and role-model behaviours of excellent managers rewarded. Thus rigorous recruitment and selection procedures, on-going education and training facilities, and clear guidelines about what is expected of each employee are needed. World-class manufacturing processes such as JIT, TQM and TPM are closely related and, in combination, can help foster the achievement of better performances.

\section{SUPPORT SYSTEMS}

Strategic initiatives, such as the introduction of multi-skilling, inter-trade flexibility, RCM, TPM, as well as optimising the redesign of work processes and structures, often fail to deliver the expected benefits. This arises because appropriate management behaviour and support 
systems (including pertinent available information and training, performance management and reward systems) that should be aligned with using these initiatives were not in place when the change programmes were implemented. Effective deployment of information technology in support of maintenance operations is increasingly a vital issue [14].

\section{PARTICIPATION AND AUTONOMY}

Participation by all personnel is part of the Japanese kaizen concept. Employee empowerment is crucial in order to create commitment in the employees' minds to this end; the management should involve all employees in defining work objectives, specifying how to achieve them and setting ambitious but realistic targets. Senge [12] maintains that workers participation must be highly valued and intrinsic motivation sought. Argyris [15] emphasised that while autonomy should be a core concept of empowerment, the management should nevertheless retain control through information systems, processes and tools. Workers must be empowered to make decisions relative to achieving high quality in the production process.

\section{TEAMS}

The use of teams is based on the old adage that" two heads are better than one". The teams meet regularly to correct problems and discuss potential improvements by using techniques such as brainstorming, discussion and quality control.

\section{EDUCATION AND TRAINING}

A greater level of skill can be achieved relatively quickly through formal training. This should not be limited to the transfer of technical skills and knowledge that are needed for optimal task performance: it should also cover generic matters like the business imperatives peculiar to the organisation (e.g. what determines the value of the product or service to the customer), problem-solving techniques, team dynamics and facilitation skills [4]. The company's ideals should be communicated to all personnel through education; everyone should be able to understand what these ideals are and why they are important for the organisation.

\section{REWARDS AND RECOGNITION}

From a study of two "best-practice" organisations (namely Fel-Pro and Steelcase), Meinoun [16] identified the following critical success factors for a reward-and-recognition process that encourages "lean" work:-

- Top management's commitment to lean work and team-based rewards and recognition

- Management being readily available and visible

- Employees are regarded as the organisation's most valuable asset.

- Employees value empowerment and involvement as a form of reward and recognition.

- The organisation relies on structured processes, policies and comprehensive, but succinct, documentation as components of (i) a continual process of learning from others, (ii) a pragmatic search for ideas, (iii) reducing time-consuming and labourintensive processes requiring external discipline, and (iv) viable tools providing useful information for improving virtually any business activity.

If an organisation stresses teamwork, the remuneration structure should promote cooperation rather than undermine it. A wide variety of remuneration programmes exist that take into 
account factors other than rank, experience and length-of-service and are used in progressive organisations, while some others use pay -for skill programmes to develop multi-skilled employees. Pay - for - performance and goal - sharing programmes award bonuses that are linked to group performances. Argyris [15] cautions that offering the "right" rewards alone is unlikely to produce sustained empowerment. The power of such methods to maintain commitment declines with use: today's privileges become tomorrow's rights. Just involvement and autonomy are the main lasting motivations that activate the human mind and drive human effort.

\section{PERFORMANCE MEASUREMENT}

In a globally - competitive economy, enterprises must pay continuous attention to increasing responsiveness to changes in customers' demands and to the achievement of a competitive advantage over their rivals. This means that the organisation should be addressing the questions of:-

- How well does it facilitate the achievement of its mission?

- How does it compare with respect to effectiveness and efficiency with other organisations?

- How does it report its achievements to its stakeholders?

- How does it get feed-back from those stakeholders?

Performance is measured with reference to clearly-defined objectives. Campbell [17] gave the commonly-classified maintenance performance indicators as measures of:

- Equipment performance, such as its availability, reliability and overall equipment effectiveness (OEE)

- Process performance, such as the ratio of planned to unplanned work, or scheduled compliance

- $\quad$ Cost performance, such as the costs for labour, material and maintenance

These performance indicators may be tracked because:-

- The indicators have been used by the organisation previously.

- Some of them are used for benchmarking in other organisations.

- The required data are easy to collect.

- Regulators or the corporate office mandates that some of them should be measured.

Simson [18] is of the opinion that these diagnostic measures determine whether the various aspects of the maintenance operations remain properly in control or compare favourably with those of counterparts elsewhere. Thus, they are used largely to support operational control and benchmarking purposes. Tsang [19] has described a process for managing maintenance performance from a strategic perspective (see figure 1). 


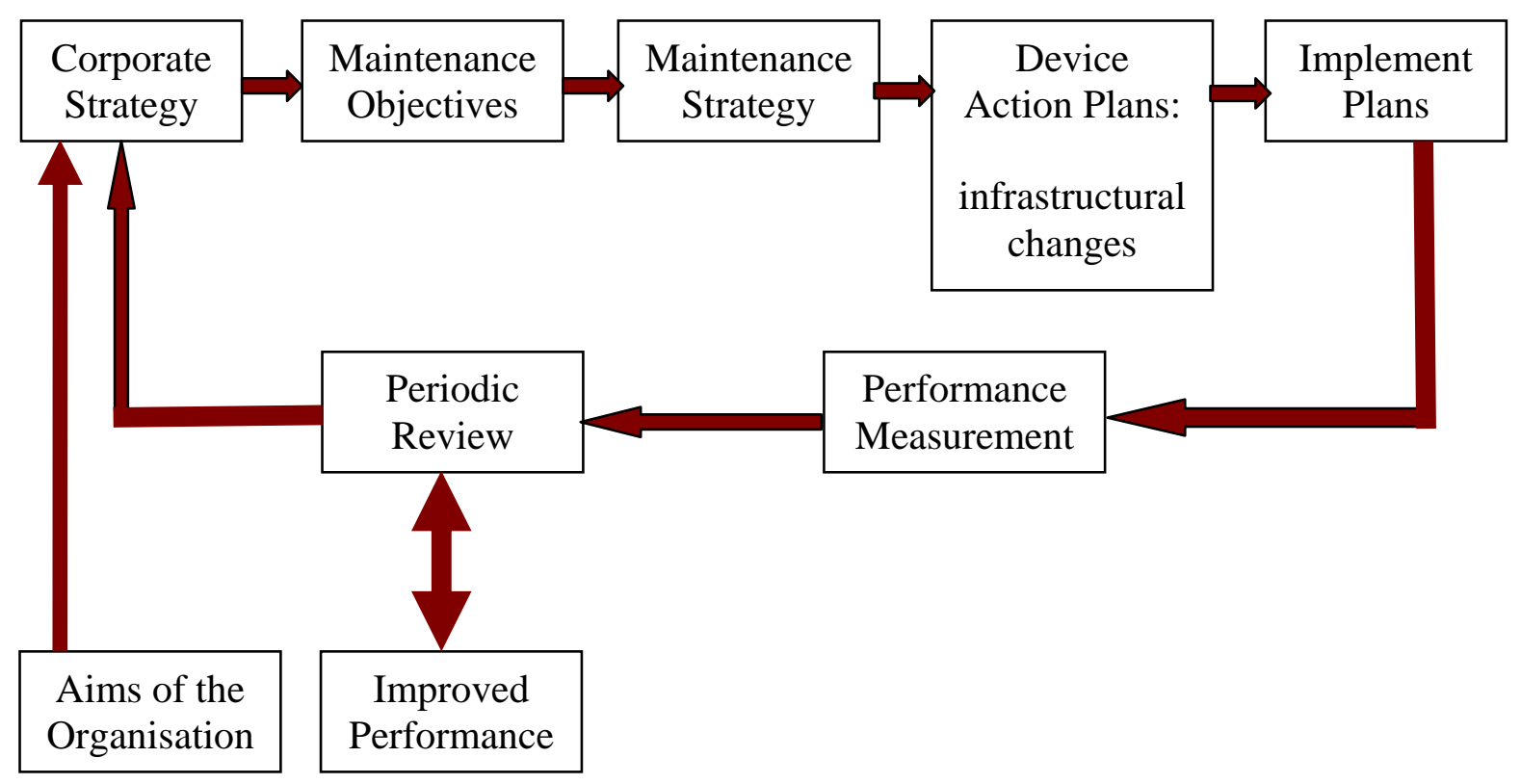

Figure 1. SMPM (after 19)

A core feature of the SMPM process is the balanced scorecard (BSC), which provides a presentation of strategic-performance measures from four perspectives (i) financial, (ii) customers, (iii) internal processes, and (iv) learning and growth [20]. By using the BSC, the strategy becomes more tangible and actionable with respect to strategic objectives, the related performance measures and their targets, and action plans. Tsang and Brown [21] reported on an electricity-generating facility's experience of introducing a BSC to measure the total performance of its maintenance functions. The BSC enables a holistic assessment of unit performance to be achieved and guards against sub-optimisation, because all the key measures that collectively determine the total performance of maintenance are monitored.

\section{BENCHMARKING}

This has become one of the most popular business-management tools of the last decade. It has been promoted as a technique that when implemented brings improvements in quality, productivity and efficiency to an organisation's business processes by learning from the reasons for other organisations' successes and the application of these better practices in one's own firm. Spendolini [22] identified activities which need benchmarking within several firms, studied best practices by others in these categories, and introduced new processes and systems to enhance productivity and quality.

Goals for a benchmarking process are to:

- Identify key performance measures for each function of a company's operations.

- Measure the internal performance levels of the company as well as those of its competitors.

- Compare performance levels in order to identify areas of competitive advantage and weakness.

- Implement programmes for closing the gaps between the internal operations success rates and those of other companies. 
Benchmarking is a process in which companies assets their achievements against those implementing best practice e.g. with respect to having:-

- A strong network for the vertical, horizontal, diagonal, intra-team and inter-team communications

- Performance-measurement criteria in place

- Employees participating enthusiastically in training

\section{COMMUNICATIONS}

In the organisation, the ability to communicate effectively is an essential skill at all levels from the front-line supervisor to the CEO. In a culture that stresses participation and autonomy, the function of the top management is not to control but to support [4], encourage and make available hands-on coaching and consultation to help employees avoid unnecessary frustration and waste of effort, to increase task- relevant knowledge and skills, and to formulate uniquely appropriate performance strategies that result in process improvements. The management should be responsive to requests from employees and ensure that the resources required for enhanced performance are available when needed. MacCoby [23] maintained that every enquiry or complaint should be considered to be an opportunity for improvement, and all the employees are encouraged to turn their complaints into improvements which, if adopted, lead to the person making the suggestion being duly rewarded.

Managers formulate strategies, make decisions and monitor progress against plans by collecting, retrieving and analysing data. Management information systems should allow a continuous rapid flow of information through the organisation to support satisfying these managerial responsibilities [4]. Many industries, during the recent period of rapid changes brought about by technological break-throughs, have improved their networking technologies in order to expand their operations globally. Advances in computer technologies are changing management philosophies: the growth of the internet and the World Wide Web are creating virtual organisations and influencing the management concepts.

The emergence of enterprise systems (ESs) - soft packages, with fully-integrated modules for controlling the major processes in the entire organisation - offers the promise of integrating all the information streams in the organisation flowchart. However, Davenport [24] maintains that the decision to introduce a generic, off-the-shelf ES has its pitfalls. Managers should check whether the logic of the process is in conflict with the logic of the organisation's practices. The suitability of an ES should be determined from a strategic perspective. In other words, the whole enterprise should be more uniformly stressed, not just one part of the process.

According to Tsang [4], if maintenance is a significant function in an organisation, the ES should include modules that have facilities for maintaining records of the history of each item of equipment used, support for preventive maintenance, work-order control, inventory control and purchasing. Through integration with other software modules, that handle payroll, accounts payable, cost accounting, shop-floor data collection and knowledge-base diagnostics, real-time decision-support information can be retrieved by managers using userfriendly interfaces.

Other requirements, imposed by the maintenance managers, include the modelling of lifetime distributions, regular inspections as part of preventive maintenance schedules, and 
equipment-replacement decisions to provide the data for the documentation of failure modes, effect and criticality analysis (FMECA). The process should support the BSC. The design should follow the logic of the process- strategic objectives that are the linked to their performance measures, which, in turn, have their respective targets: the top-level BSC is cascaded down to lower level ones.

\section{E-MAINTENANCE}

A major aspect of maintenance management is the optimisation of the inspection, maintenance and replacement decisions [14]. These depends on the availability of good quality and timely data, captured by the various computerised systems in the supplier, user and service- provider organisations. The volume of data available to maintenance managers is expanding rapidly. The problem is exacerbated in the case of organisations with operations covering large geographical areas, such as public utilities, mining and transportation. Thus the potential of digital technologies offers new options to deal with the resulting vat amount of data. Two types of e-maintenance initiatives [4] have evolved:-

- e-CBM. Remote sensing devices are deployed to monitor the condition and performance of each physical asset. The captured data are transmitted to a secure site for analysis and decision-making.

- e-CMMS. The web enables computerised maintenance management systems (CMMSs) to be used. The entire application is available over the web, through the application service provider (ASP). The expert ASP knows the user's software requirements and provides the user with a total solution and hence the driving forces that makes this option increasingly appealing [13]. In the e-maintenance model, internal processes are expanded to cover other applications on the web.

\section{CONCLUSION}

Organisations that are successful at implementing employee-involvement practices use a strategy to achieve a match between the practices and the organisation's culture (i.e., its unique values, beliefs and mission). Today's competitive environment requires that industries succeed in sustaining full production capabilities, while minimizing capital investment. From a maintenance perspective, this means maximizing equipment reliability (i.e. achieving maximum uptime), while extending the plant's life. This proactive approach needs a total planned quality maintenance (TPQM) programme, the systemising of all (i.e. preventive, predictive, and planned) maintenance, plus the control of maintenance quality. As the global marketplace becomes more competitive, such advantages are necessary in order to maintain the long-term corporate economic health. The strategic dimensions of maintenance management form the basis for wise decision-making which can be performed during any phase of the system's life, but probably sooner than later. Organisational design, maintenance methodology, adequate support systems, corporate culture and general management have been identified as necessary factors for the effective maintenance in Nigerian electric-power stations. The relevant factors are human-data information flow and having an excellent learning organisation. Tsang [4] maintains that understanding the behaviour of personnel at work as well as the conditions for enhancing group effectiveness will produce superior organisational designs that stimulate people's minds and create an internal commitment to the

enterprise. Due consideration of these factors will also increase the chances of success when faced with rapid change. The maintenance strategy should contain considerations of the following elements: 
- Maintenance organisation and management

- Measures of effectiveness

- Work control

- Maintenance-management information system

- Personnel records regarding competences

- Technical documentation

- Logistic support

- Maintenance tasks

- Maintenance engineering.

The seamless flow of information is another enabler for achieving exemplary performance. The availability of pertinent information can influence a person's behaviour when a performance-management process (communicating the espoused strategy and emphasizing a balanced assessment) is employed [4]. E-maintenance has tremendous potential for implementing a maintenance strategy. However, the characteristics of a world-class plantpreventive maintenance, with no machine breakdowns during scheduled runs, are as yet virtually non-existent in Nigeria.

\section{REFERENCE}

1. Moubray, J (2000), The Case against Streamside RCM, Aladon Ltd, Ashville, N. Carolina.

2. Moubray, J. (1995), Maintenance Management a new paradigm. Aladon Ltd. Ashville, N. Carolina.

3. Saranga, H. (2002), "Relevant condition parameter strategy for an effective conditionbased maintenance, Journal of Quality in Maintenance Engineering, Vol. 8 No1, pp92 -105 .

4. Tsang, A.H. C., (2002), Strategic dimensions of maintenance management, Journal of Quality in Maintenance Engineering, Vol.8 No1, pp.7-39.

5. Kennedy, R. (2002) Examining the processes of RCM and TPM. Centre for TPM, Australia

6. Taylor, J.C and Felton, D.F (1993), Performance by Design: Sociotechnical Systems in North America, Prentice-hall, Englewood cliffs, NJ.

7. Holodny, H. and Stjeinberg, T (1993), "Self-managing teams: the new organisation of work”, in Cohen, A.R (Ed.), The portable MBA in management, John Wiley, pp.279314

8. Wilkins, R.H. (1994), The Quality-Empowered Business, Prentice-Hall, Englewood Cliffs, NJ. Pp367 -369.

9. Hyland, P. and Beckett, R (2002) Learning to compete: the value of internal benchmarking, Benchmarking: an International Journal, Vol. 9 No3 pp. 393-304. Also Organisational learning”, Journal of Management Studies, Vol. 36 No7, pp.897919.

10. Drew, A.W (1997) From knowledge to action: the impact of benchmarking on organisational performance, Long-range Planning, Vol. 30 No.3, pp.427-41. 
11. Harvey, C. and Denton, J. (1999), “To come of age: the antecedents of organizational learning. Journal of Quality in Maintenance Engineering, Vol. 1, No. 3. pp 18 - 24.

12. Senge, P.M (1999), "It's the learning: the real lesson of the quality movement", Association for quality November/December, pp.34-40.

13. Day, M (1994) Can organisations have a learning disability? Canadian Manager, Summer, pp. 21-31

14. Tsang, A.H.C, Jardine, A.K.S, Campbell, J.D and Picknell, J.V. (2000) ReliabilityCentred Maintenance: a key to maintenance excellence, City University of Hong Kong, China.

15. Argyris, C. (1998), Empowerment: the emperor's new clothes, Harvard Business Review, Vol. 76 No3 pp 98-105.

16. Meinoun, E. (1995), “A rewarding experience”, The Benchmark, February, pp.40-2.

17. Campbell, J.D. (1995), Outsourcing in maintenance management: a valid alternative to self provision, Journal of Quality in Maintenance Engineering, Vol.1 no3, pp.18-24.

18. Simson, R. (1995), Levers of control. How managers use innovative control systems to drive strategic renewal, Harvard Business School press, Boston, MA; also in Society, the Total Quality Magazine, Vol 12, No 3, pp172-8

19. Tsang, A.H.C (1998), “A strategic approach to managing maintenance performance Journal of Quality in Maintenance Engineering, Vol. No. 2, pp.3-17.

20. Kaplan R.S and Norton, D. P. (1996) The Balanced Scorecard, Harvard Business School Press, Boston.

21. Tsang, A.H.C Brown W.L (1999), Managing the maintenance performance of an electric utility through the use of balanced scorecards, New Engineering Journal, Vol.2 No3, pp.22-9.

22. Spendolini, M.J. (1992), The Benchmarking Book, AMACOM. NY.

23. MacCoby, M (1997), "Is there a best way to build a car?", Harvard Business Review, Vol. 75 No6, pp.161-71

24. Davenport, T. H (1998), Putting the enterprise into the enterprise system, Harvard Business Review, Vol. 73 No.3 pp. 94-102. 